Memorias del VII Encuentro Nacional de Experiencias en la Enseñanza de la Biología y la Educación Ambiental y II Congreso Nacional de Investigación en la Enseñanza de la Biología

\title{
APROXIMACIÓN A LAS CONCEPCIONES ACERCA DE CÉLULA DE ESTUDIANTES DE SEXTO GRADO DEL INSTITUTO NACIONAL DE EDUCACIÓN MEDIA INEM JULIAN MOTTA SALAS DE NEIVA
}

\section{APPROACH TO THE CELL CONCEPTS OF SIXTH GRADE STUDENTS OF THE INSTITUTO NACIONAL DE EDUCACION MEDIA INEM JULIAN MOTTA SALAS OF NEIVA}

\author{
John Wilmar Arce Zuñiga ${ }^{1}$ \\ Elías Francisco Amórtegui Cedeño ${ }^{2}$
}

\section{Resumen}

A continuación presentamos resultados preliminares del desarrollo de práctica pedagógica I al interior del Programa de Licenciatura en Ciencias Naturales y Educación Ambiental de la Universidad Surcolombiana (Neiva-Colombia) llevada a cabo en el Instituto Nacional de Educación Media INEM Julián Motta Salas. El grupo de trabajo está conformado por estudiantes de sexto grado cuyas edades oscilan entre 10 y 13 años. Para el caso de esta ponencia nos referimos particularmente a la aproximación a las concepciones acerca del concepto célula que tienen los estudiantes. La metodología está enmarcada en una perspectiva cualitativa, empleando el análisis de contenido y haciendo uso del cuestionario. Los resultados muestran 3 grandes tendencias en la que se haya el componente cuerpo humano, plantas y animales y un grupo que hace referencia a conceptos muy diversos al cual hemos llamado asociaciones variadas. En donde la tendencia relacionada con el cuerpo humano es la mayoritaria implicando que los estudiantes conciben la célula como una característica exclusiva de los seres humanos. Finalmente, mostramos algunas implicaciones en la enseñanzaaprendizaje de las Ciencias Naturales y proyectamos algunas posibles estrategias para favorecer al aprendizaje del concepto.

\section{Abstract}

Here are preliminary results of the development of teaching practice I into the Bachelor of Education Program with Emphasis on Basic Science and Environmental Education at the University Surcolombiana (Neiva-Colombia) held in an Educational Institution Officer. The working group is comprised of sixth-grade

\footnotetext{
${ }^{1}$ Estudiante de octavo semestre del Programa de Licenciatura en Educación Básica con Énfasis en Ciencias Naturales y Educación Ambiental. Universidad Surcolombiana. jwarce@gmail.com

2 Docente de Planta Tiempo Completo. Programa de Licenciatura en Educación Básica con Énfasis en Ciencias Naturales y Educación Ambiental. Universidad Surcolombiana. Magister en Educación. elias.amortegui@usco.edu.co
} 
Memorias del VII Encuentro Nacional de Experiencias en la Enseñanza de la

Biologia y la Educación Ambiental y II Congreso Nacional de Investigación en la Enseñanza de la Biología

students aged between 10 and 13 years. In the case of this paper we refer particularly to the approach to the conceptions about the cell concept for students. The methodology is framed in a qualitative perspective, using content analysis and using the questionnaire. The results show three major trends in which the component is human body, plants and animals and a group that refers to very different concepts which have called associations varied. Where the trend related to the human body is the majority implying that students conceived the cell as a unique characteristic of human beings. Finally, we show some implications for teaching and learning of Science and project some possible strategies to promote the learning of the concept.

Palabras Clave: Célula, Concepciones, Ciencias Naturales y Educación Ambiental.

Keywords: Cell, Concepts, Science and Environmental Education.

\section{Introducción}

El Programa de Licenciatura en Ciencias Naturales y Educación Ambiental, es un programa de formación inicial de docentes adscrito a la Facultad de Educación de la Universidad Surcolombiana (Neiva-Huila); éste, consta de nueve semestres y cuatro ámbitos de formación (Biología, Química, Física y Didáctica) que componen el núcleo común dentro del componente básico específico en el plan de estudios. Al interior del programa, se realizan dos prácticas pedagógicas llevadas a cabo al interior de octavo y noveno semestre respectivamente, realizándose la Práctica Pedagógica I en el nivel de la básica secundaria ${ }^{3}$ en una institución educativa de cualquier municipio del Departamento del Huila, en el área de Ciencias Naturales y Educación Ambiental; mientras que la Práctica Pedagógica II se lleva a cabo en la Educación Media, particularmente en el área de Química o Física.

Para el caso de ambas prácticas pedagógicas, tienen una duración de dieciocho semanas, de las cuales la segunda corresponde al proceso de observación del grupo de estudiantes por parte del futuro docente, en donde examina la dinámica de la clase teniendo en cuenta aspectos como los gustos, intereses, experiencias, ideas previas, dificultades de aprendizaje de los estudiantes, entre otros; las siguientes dieciséis semanas son de intervención directa junto con el estudiantado por parte del futuro docente, en donde pone en juego los conocimientos adquiridos en su formación inicial.

${ }^{3}$ De acuerdo a la normatividad vigente del Ministerio de Educación Nacional. 
Memorias del VII Encuentro Nacional de Experiencias en la Enseñanza de la

Biologia y la Educación Ambiental y II Congreso Nacional de Investigación en la Enseñanza de la Biología

Para el caso de la primer semana, el practicante futuro docente realiza una previa planificación junto con el coordinador de práctica pedagógica del programa, sus compañeros y un docente asesor quien cuenta con amplia formación en investigación educativa, pedagógica y didáctica, teniendo en cuenta aspectos tales como: el modelo didáctico que orienta la práctica pedagógica, los requerimientos y requisitos de la institución educativa al igual os contenidos dentro del plan de estudios del curso a intervenir, las finalidades de la Práctica Pedagógica, las posibles estrategias a implementar de acuerdo a las bases teórico-pedagógicas que se tienen, Criterios y mecanismos de Evaluación, actividades CTS-A, uso de TICs, entre otros.

Para el caso de Colombia, las directrices planteadas por el Ministerio de Educación Nacional, contemplan dentro de los Estándares y Competencias para los grados sexto y séptimo "...Explico la estructura de la célula y las funciones básicas de sus componentes..." es por esto que para el caso de esta ponencia nos referimos a este concepto.

Con relación a los antecedentes cabe destacar que por espacio no los presentamos, sin embargo para el caso del Departamento del Huila y la Ciudad de Neiva no existen trabajos sobre la aproximación a las concepciones que tienen los estudiantes sobre célula ni sobres las implicaciones de estas en la enseñanzaaprendizaje de las Ciencias Naturales.

Para el caso de esta ponencia, definimos las concepciones tal como plantea Amórtegui (2011) como sistemas de ideas que tienen los sujetos que pueden evolucionar a través de un proceso de restructuración que puede o no ser consciente, basado en la interacción con otras ideas y experiencias de los sujetos, además las concepciones originadas en un contexto particular que provocan un aumento en su grado de complejidad no se transfieren de manera automática y mecánica a otros contextos y problemas de la misma clase, pero si pueden influir en ellos, están fuertemente arraigadas en la medida que son coherentes, flexibles y funcionales $\mathrm{y}$, posibilitan explicaciones causales a fenómenos físicos y al igual que las rutinas, son resistentes al cambio y consecuentemente, pueden constituir obstáculos para la transformación. Para el caso de esta ponencia cabe resaltar que nos referimos exclusivamente a las respuestas textuales de los estudiantes a partir de la aplicación de un cuestionario.

Específicamente en el campo de la enseñanza-aprendizaje de la Biología, las concepciones son fundamentales debido a que los estudiantes tienen explicaciones sobre los fenómenos biológicos que difieren de los aceptados desde el punto de vista científico, formadas de formas espontánea como consecuencia de sus percepciones sobre los fenómenos naturales (Banet, 2000). 
Memorias del VII Encuentro Nacional de Experiencias en la Enseñanza de la

Biología y la Educación Ambiental y II Congreso Nacional de Investigación en la Enseñanza de la Biología

El estudio se llevó a cabo desde un enfoque cualitativo, empleando el método de análisis de contenido en el proceso de sistematización, y usando como herramienta de recolección de información el cuestionario, el cual contenía como preguntas abiertas "Para ti, ¿qué es una célula?, ¿Qué tipo de cosas crees que poseen células?, y representa en un dibujo una célula". El grupo objeto de estudio consistió en 36 estudiantes del grado 601 de la jornada de la mañana cuyo rango de edades oscila entre 10 y 13 años pertenecientes en su mayoría a los estratos socioeconómicos 1 y 2.

\section{Resultados Y Discusión}

Los hallazgos permitieron establecer 3 grandes tendencias sobre las concepciones de los estudiantes. A continuación presentamos las características de cada una, así como su frecuencia y en algunos casos mostramos evidencias textuales y fotográficas de las respuestas de los estudiantes. Las tendencias que se dieron teniendo en cuenta el número de estudiantes que hicieron parte de cada una de ellas fueron las siguientes:

Cuerpo humano: En esta tendencia mayoritaria (22 estudiantes), los alumnos relacionan la célula exclusivamente con el cuerpo humano en tanto hace parte de él y en grandes cantidades; sin embargo no explicitan las características, funciones, importancia y roles que cumple como unidad estructural, funcional y de regulación de los sistemas vivientes, además de que no involucran ningún otro tipo de organismos tales como microorganismos, plantas, hongos, entre otros.

E1.P1.1 "Pequeña cavidad, organismo elemental que contiene el cuerpo humano"

E13.P1.1 "La célula es una pequeña cosa pero dentro de nuestro cuerpo hay un poconón".

Lo anterior implica por una parte un obstáculo en el aprendizaje de los estudiantes sobre el conocimiento biológico, en tanto sus ideas se circunscriben en una visión antropocéntrica de la Biología, en la que el ser humano prima frente a las otras especies. También muestra la dificultad en el reconocimiento de la biodiversidad de organismos que existen en el planeta y más aun en un contexto rural en el que se encuentran inmersos los estudiantes; situación muy similar con estudios reportados tal como el de Yen, Yao y Mintzses (2007). De acuerdo a Banet (2000) es fundamental que los estudiantes adquieran un aprendizaje sobre los seres vivos de tal forma que les permita dar cuenta de la estructura celular y sobre pasar la concepción errónea sobre el ámbito biológico de la teoría celular en la que los alumnos no conciben que todos los órganos estén compuestos de células. 
Memorias del VII Encuentro Nacional de Experiencias en la Enseñanza de la

Biología y la Educación Ambiental y II Congreso Nacional de Investigación en la Enseñanza de la Biología

Por otra parte, esta concepción mayoritaria podría llevar a los estudiantes a que tengan la idea de que las células se restringen a los animales en general, tal como ha reportado Jiménez (2003).

Asociaciones variadas: Para este segundo grupo del cual corresponden 11 estudiantes, se puede observar como los chicos hacen referencia a la célula desde diferentes comparaciones creando su propio concepto y al mismo tiempo hablan de la célula como algo que no logran distinguir y que simplemente se refieren a ella en términos de un objeto, cantidad, forma, un "algo" o una "parte" sin llegar a una concepción concreta de lo que puede llegar a ser una célula, llevándola a hacer parte de un conjunto o como algo que sirve favorablemente dentro de un organismo, ya sea la reparación o el funcionamiento del mismo. De igual forma hubo unas pocas excepciones en donde un número muy reducido de estudiantes (3) habló de la célula haciendo alusión a un concepto de carácter más elaborado como organismo.

\section{E18.P1. "Es por la cual a nosotros nos funcionan los órganos".}

E12.P1. "Es una cosa diminuta que ayuda al organismo"

Aquí se puede observar las falencias que presentan los chicos en el momento de elaborar un concepto claro acerca de célula, sus conceptos previos o lo que le enseñaron en la escuela primaria acerca del concepto célula no es muy claro ya que presentan bastante confusión a la hora de dar una definición. Se nota que de la escuela primaria al colegio hay una especie de choque en cuanto al manejo de este concepto, sería bueno que los chicos pudieran ingresar al colegio desde la escuela haciendo "puente" en cuanto al manejo de este tipo de conceptos que se manejan durante toda la preparación académica en el campo de la Biología y que son de gran importancia para evitar cambios conceptuales fuertes y que al contrario fueran más familiarizados para ellos. Además se nota a nivel estructural la incapacidad de los alumnos de dar cuenta de distintos clases de células, pues tal como se observa en la Imagen 1, confunden estructuralmente los glóbulos blancos, los glóbulos rojos y en general una célula ideal. 
Bio-grafía Escritos sobre la Biologia y su Enseñanza.

Edición Extra-Ordinaria. ISSN 2027-1034 P. p 263 - 270

Memorias del VII Encuentro Nacional de Experiencias en la Enseñanza de la Biología y la Educación Ambiental y II Congreso Nacional de Investigación en la Enseñanza de la Biología

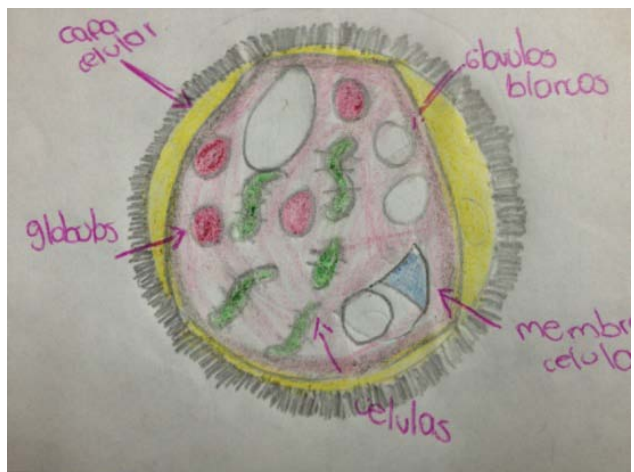

Imagen 1. Fotografía sobre un dibujo acerca de célula de un estudiante de sexto grado.

Plantas y animales: La tercera tendencia en la cual estuvieron involucrados sólo dos estudiantes, fueron los únicos que relacionaron la célula con la flora y la fauna, en esta parte se podría decir que se sale un poco de la mirada antropocéntrica que tienen la mayoría de los estudiantes con respecto a la célula.

E2.P1. "Es una pequeña cavidad, organismo elemental que compone el cuerpo de las plantas y animales".

E21.P1. "Es un organismo que procede sobre los seres bióticos como la flora y la fauna".

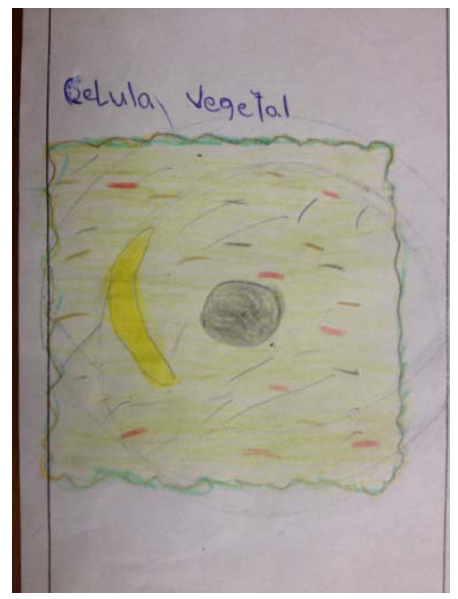

Imagen 2. Fotografía sobre un dibujo acerca de célula de un estudiante de sexto grado. 
Memorias del VII Encuentro Nacional de Experiencias en la Enseñanza de la

Biología y la Educación Ambiental y II Congreso Nacional de Investigación en la

Enseñanza de la Biología

Esta tendencia, aunque en un porcentaje muy bajo nos muestra que los chicos pueden asociar la célula como una unidad que conforma a otros organismos vivos, en este caso tratándose de las plantas y lo animales; pero de igual forma al ser tan pocos los estudiantes que se refieren a esta parte, se puede deducir que la célula está más enfocada hacia una sólo temática y que muy poco lo generalizan con el resto de organismos, lo cual permite trascender de las ideas erróneas que han mostrado Banet (2000) y Jiménez (2003).

\section{Reflexiones sobre la enseñanza aprendizaje del concepto}

Según Mortimer, (2000); más que hacerles ver a los estudiantes que se encuentran en un error, hay que poner en manifiesto que hay interpretaciones distintas que fueron aceptadas en otro tiempo y además el docente debe estar en la capacidad de plantear problemas que puedan ser resueltos en contextos variados como los relacionados con animales y plantas, vertebrados e invertebrados, cuestiones anatómicas, cambios de tamaño, forma, color y de tipo fisiológico; también debe estar en la capacidad de indagar, movilizar, hacer entrar en conflicto y favorecer el enriquecimiento de las concepciones de los estudiantes (Amórtegui, 2011).

Teniendo en cuenta la imposibilidad de que los estudiantes den cuenta de elementos micro, cuando nunca han tenido la posibilidad de observarlos al microscopio y que además esto se convierte en una dificultad de aprendizaje (Gagliardi, 1986) y en un obstáculo epistemológico, es fundamental el trabajo de laboratorio que permita a los estudiantes reconocer las estructuras celulares y desde allí la comprensión de la teoría celular.

Por último, consideramos que de acuerdo a Valbuena (2007) la enseñanza de la Biología debe estar centrada en problemas socialmente relevantes que permitan ser situaciones motivantes y llamativas para los estudiantes en aras de generar en ellos actitudes hacia la ciencia, procedimientos de indagación y la comprensión de conceptos, que para el caso de la región del Huila y la ciudad de Neiva puede ser la prevalencia de enfermedades y por ejemplo el alto uso de sustancias psicoactivas en la población juvenil y en edad de escolarización.

\section{Conclusiones}

Durante el proceso de indagación sobre el concepto de célula en los estudiantes de sexto grado se pudo determinar que en un alto porcentaje el concepto se maneja dentro de un marco que está determinado hacia el componente cuerpo humano, haciendo énfasis en que estas no solo hacen parte de él, sino que componen cada uno de nuestros órganos y de igual forma lo relacionan con el proceso de reparación. Aunque se pueden hallar respuestas relevantes con cierto grado de elaboración estructural, esta tendencia muestra las falencias de los 
Memorias del VII Encuentro Nacional de Experiencias en la Enseñanza de la

Biología y la Educación Ambiental y II Congreso Nacional de Investigación en la Enseñanza de la Biología

estudiantes en cuanto a relacionar la célula solo con su cuerpo y no con diversos grupos de organismos vivos existentes.

Se puede apreciar como los estudiantes no pueden en gran medida elaborar un concepto más concreto con respecto a la célula, sino que manejan algún tipo de analogía, comparándola con otras cosas y una funcionalidad, pero sin determinar que puede llegar a ser una célula para ellos.

Muy pocos estudiantes tuvieron la noción de poder asociar la célula con otros grupos de seres vivos como los animales y las plantas, lastimosamente fueron muy pocos, fue la tendencia con menor porcentaje, lo cual indica como los chicos desde la escuela no se les ha enfatizado en la célula como componente estructural no solo del cuerpo humano sino de una gran diversidad de organismos vivos.

\section{Bibliografía}

- Amórtegui, E (2011). Concepciones sobre prácticas de campo y su relación con el conocimiento profesional del profesor, de futuros docentes de Biología de la Universidad Pedagógica Nacional. Tesis para optar al título de Magíster en Educación. Bogotá DC: Universidad Pedagógica Nacional.

- Banet, E (2000). Enseñanza aprendizaje del conocimiento Biológico. En: Perales, F \& Cañal, P (2000). Didáctica de las ciencias experimentales. Madrid: Acoy.

- Gaglardi, R (1986). Los conceptos estructurales en el aprendizaje por investigación. Enseñanza de las ciencias. 4 (1). Pp 30-35.

- Jiménez, M (2003). La enseñanza y el aprendizaje de la Biología. En: Jiménez, $\mathrm{M}$ et al (2003). Enseñar Ciencias. Barcelona: Graó.

- Mortimer, E.F (2000): Linguagem e formacao de conceitos no Ensino das Ciencias. Belo Horizonte. Editora UFMG. (Traducido al castellano, ed. Antonio Machado, en prensa.

- Valbuena, E. (2007). El Conocimiento Didáctico del Contenido Biológico. Estudio de las concepciones disciplinares y didácticas de futuros docentes de la Universidad Pedagógica Nacional (Colombia). Tesis para optar al título de Doctor en Didáctica de las Ciencias Experimentales. Universidad Complutense de Madrid. 